\title{
Altersgemäß Lernen: Die Rolle von Lernfitnesstrainings
}

\author{
CHRISTIAN STAMOV-ROßNAGEL, KLAUS-W. WEST
}

Es gibt seit einigen Jahren einen Diskurs darüber, wie Beschäftigte alters- und alternsgemäß lernen und arbeiten können. Spitzenvertreterinnen und -vertreter der Politik, der Arbeitgeberverbände, der Gewerkschaften und der beiden christlichen Kirchen engagieren sich für dieses Thema; Bundes- und Landesministerien fördern mit großen Summen Projekte zur Entwicklung des Lernens und der Weiterbildung in der Arbeit.

Der heutige Diskurs für eine bessere betriebliche Inklusion älterer Beschäftigter knüpft an das Leitbild und die Aktivitäten zur „Humanisierung der Arbeit“ an. Einstmals vom damaligen Bundesforschungsminister Hans Matthöfer initiiert, gab es von 1974 - 1989 ein Programm der Bundesregierung zur „Humanisierung des Arbeitslebens (HdA)“, das zum Ziel hatte, die Arbeitsinhalte und -beziehungen zu verbessern und belastende und gesundheitsgefährdende Arbeitssituationen abzubauen. Es wurde 1989 vom Forschungs- und Entwicklungsprogramm „Arbeit und Technik“ abgelöst. 2001 folgte das Programm „Innovative Arbeitsgestaltung - Zukunft der Arbeit" des Bundesministeriums für Bildung und Forschung. Im Mittelpunkt stand hier die Erforschung zukünftiger Formen der Arbeit.

Der gegenwärtige Diskurs ist maßgeblich durch einen der Megatrends unserer Zeit bestimmt: den demografischen Wandel. Wenn es darum geht, das Lernen und die Weiterbildung der Beschäftigten in der Arbeit zu fördern und die Arbeitsplätze lernförderlich zu gestalten, so ist es wichtig, sich die Humanisierungsanstrengungen der Vergangenheit zu vergegenwärtigen. Dies hilft zu klären, in welcher Situation man sich derzeit befindet.

Dazu gehört, anzuerkennen, dass der demografische Wandel in der Gesellschaft, in den Unternehmen, in öffentlichen Verwaltungen und Einrichtungen und in der Dienstleistungsarbeit angekommen ist. Der Anteil älterer Beschäftigter wächst - ein Trend, der sich nicht zuletzt infolge veränderter sozialpolitischer Rahmenbedingungen künftig weiter verstärken wird. Das aber verändert auch die „Bedeutung des Alters“. Eine ganze Reihe von Themen, zu denen auch die Lern- und Leistungsfähigkeit älterer Beschäftigter gehört, beschäftigt heute die Menschen. ${ }^{1}$

Wir gehen davon aus, dass wir gegenwärtig eine „Revolutionierung der Altersordnung “ in unserer Gesellschaft erleben. Arbeit und ein „tätiges Leben“ (Hannah Arendt) verändern sich, wenn die Lebenserwartung der Menschen steigt und sich das Alterszentrum der Gesellschaft nach oben verschiebt. Mit der „Revolutionierung der Altersordnung" ist eine problematisch gewordene Situation in der Gesellschaft entstanden, die ein breites Spektrum von Antworten erfordert.

Klärungsbedürftig ist u. a., wie die Bedingungen der Arbeit beschaffen sein müssen, damit Menschen gesünder arbeiten können, und auch länger. Die Antwort ist für alle Beschäftigten von großem Interesse. Denn es gibt Chancen, Belastungen einzuschränken, die Arbeit abwechslungsreicher zu gestalten und Arbeit und Leben in eine Balance zu bringen. Auf diesem Wege können Win-win-Situationen geschaffen werden: für die Beschäftigten ein Zugewinn an Sinn, Anerkennung und Einkommenschancen, für die Unternehmen ein Plus an effektiver und effizienter Arbeit.

\section{Altersgemäßes Lernen}

Wie eine - auch im Sinne des lebenslangen Lernens - lernförderliche Arbeitsumgebung beschaffen sein muss, war die Schlüsselfrage eines Projektes der Jacobs University Bremen zur „altersdifferenzierten Kompetenzförderung“, das in Kooperation mit der Chemie-Stiftung Sozialpartner-Akademie (CSSA) in Wiesbaden bei der Michelin AG, Werk Bad Kreuznach durchgeführt wurde. ${ }^{2}$ Beim Reifenhersteller Michelin in Bad Kreuznach sind mehr als 1500 Menschen beschäftigt. Ziel war es, die Lernkompetenzen von Beschäftigten $\mathrm{zu}$ fördern, die nicht $\mathrm{zu}$ den intrinsisch motiviert Lernenden gehören. Es handelte sich um überwiegend ältere, lernentwöhnte Beschäftigte.

Dieses Projekt wies eine Besonderheit auf: Es wurde von Unternehmensleitung und Betriebsrat konzertiert. Beide teilten ein sozialpartnerschaftliches Kooperationsverständnis, und dies hatte erhebliche praktische Vorteile. Die Beschäftigten wussten, dass das Projekt nicht allein auf Initiative der Arbeitgeber stattfand, sondern dass der Betriebsrat es von Anfang an unterstützte. Bei einem Vertrauensthema

1 Vgl. das Leitbild für „altersgemäße Arbeit" der ChemieStiftung Sozialpartner-Akademie (CSSA) vom August 2013, http://www.cssa-wiesbaden.de/pdf-downloads.html.

2 Von April bis September 2011 führte Christian StamovRoßnagel vom Jacobs Center on Lifelong Learning (JCLL) an der Jacobs University Bremen dieses Projekt durch. 
wie „Lernen“ wurde damit die Basis für eine unvoreingenommene Beteiligung und aktives Engagement gelegt.

\section{Die Ausgangslage}

Das Projekt begann mit einer quantitativen Umfrage in zwei Produktionsbereichen. Sie ermöglichte einen Überblick über das betriebliche Lernumfeld und die individuelle Lernkompetenz der Beschäftigten. Ziel hierbei war, Ansatzpunkte für die Entwicklung eines Lernfitnesstrainings zu identifizieren, das auf die Bedarfe der Beschäftigten abgestimmt ist.

An der Befragung beteiligten sich 142 Beschäftigte aus Verwaltung und Produktion im Alter von 18 bis 65 Jahren. Der Altersdurchschnitt lag bei 41,1 Jahren. Relativ hoch war der Anteil mit Beschäftigten ohne Berufsabschluss. Aus ihren Reihen wurden in der späteren Projektphase die meisten der Trainingsteilnehmer rekrutiert.

In der Umfrage standen zwei zentrale Einflüsse auf die Lernkompetenz der Beschäftigten im Mittelpunkt. Bei Michelin gibt es lernförderliche Betriebsvereinbarungen, die Themen wie die Regelungen zur Freistellung von der Arbeit für Weiterbildungen betreffen. In Ergänzung dazu fragten die Forscher vom JCLL nach zwei Faktoren, die nur selten betrachtet werden, obwohl sie für die Lernkompetenz eine große Rolle spielen: Dies sind einerseits unternehmensspezifische Faktoren, die das Lernumfeld betreffen: das Lernklima und die Unterstützung der Beschäftigten durch Führungskräfte. Andererseits gibt es auch personengebundene Faktoren, wie z. B. die individuelle Lernkompetenz. Sie bestimmt wesentlich, wie effektiv und effizient Beschäftigte sich weiterbilden. Im Arbeitsalltag spielen beide Faktoren zusammen. Wie nachhaltig die Beschäftigten ihre Lernkompetenz auf- und ausbauen und nutzen, hängt entscheidend vom Lernumfeld ab.

Das Lernklima erfasst die von Beschäftigten wahrgenommene Unterstützung für berufsbezogenes Lernen. Dieses Klima wird abteilungsübergreifend durch für die Gesamtorganisation geltende Größen geprägt: z.B. die Bewertung der von der Firma bereitgestellten Trainingsund Ausbildungsprogramme. Hinzu kommen abteilungsspezifische Größen. In der Regel sind dies von den direkten Vorgesetzten ausgehende Einflüsse. Dazu gehören die Berücksichtigung individueller Lernbedarfe bei der Gewährung von und Ermunterung zur Weiterbildung.

Die Umfrage erbrachte vier wichtige Hinweise, die ein günstiges Lernklima bei Michelin belegen:

- $\quad$ 83,5\% der Befragten stimmten der Aussage weitgehend oder voll zu, bei Michelin herrsche die Überzeugung, dass beständiges Lernen für gute berufliche Leistung wichtig sei.

- $\quad 64,2 \%$ der Befragten stimmten der Aussage überwiegend oder voll zu, dass den Beschäftigten die Hilfsmittel zur Verfügung gestellt werden, die für Erwerb und Nutzung neuer Kenntnisse nötig sind.

- $\quad$ 71,7 \% teilten weitgehend oder vollständig die Meinung, dass Führungskräfte die Möglichkeiten zur Weiterbildung auf die Bedürfnisse der Beschäftigten abstimmen.
73,2\% waren überwiegend oder vollauf der Auffassung, dass sie von ihren Vorgesetzten zu unabhängigem und innovativem Denken ermuntert werden. Dies fördere das Vertrauen in die eigene Lernfähigkeit und erhöhe die Bereitschaft zum Lernen.

Die Umfrage zeigte weiterhin, dass - im Einklang mit dem positiven Lernklima - die Befragten dem Lernen gegenüber aufgeschlossen sind, was per se eine gute Grundlage für das lebenslange Lernen ist. Bei keiner der erfragten Einstellungen trat ein signifikanter Unterschied zwischen Beschäftigten der Altersgruppen bis 45 Jahre und über 45 Jahre zutage. So waren beispielsweise 85,3\% der Befragten der Ansicht, dass Ältere nicht weniger lernen müssten, nur weil sie viel Erfahrung besitzen. Passend dazu stimmten nur 5,9\% der Befragten der Aussage ganz oder überwiegend zu, dass in die Förderung jüngerer Arbeitnehmer mehr investiert werden sollte als in die Förderung älterer Beschäftigter.

Es zeichnete sich aber Unterstützungsbedarf ab. 36,8\% waren der Ansicht, Ältere könnten nicht mehr so gut lernen, weil das Gedächtnis nachlasse. Eine solche Einschätzung, die durch wissenschaftliche Befunde widerlegt wird, kann das Lernengagement dämpfen. 42,7 \% äußerten die Ansicht, dass ihnen beim Lernen umfassende Anleitung wichtig sei.

\section{Das Lernfitnesstraining}

Die Umfrage bildete die Grundlage für die Gestaltung des Lernfitnesstrainings. Am Training nahmen 57 Beschäftigte aus der Produktion teil, aufgeteilt in vier Gruppen, die je eine 90-minütige Trainingseinheit pro Woche in vier aufeinanderfolgenden Wochen absolvierten.

Die Trainings hatten zum Ziel, die Lernkompetenz zu steigern und die Weiterbildungsmotivation nachhaltig zu stärken. Der Trainingsansatz sah die Veränderung des individuellen Lernmodells vor. Dieses Modell wird von den Lerneinstellungen geprägt, von der Lernmotivation im Sinne einer subjektiven Kosten-Nutzen-Analyse und von den zur Verfügung stehenden Lerntechniken. Das Lernmodell bestimmt das Lernverhalten und die Nutzung der individuellen Lernkompetenz. Zwei Aspekte der Umfrageergebnisse lieferten Anhaltspunkte, in welcher Hinsicht die Lernmodelle der Veränderung bedurften. Erstens wies ein Großteil der Befragten nur geringes Wissen über Lerntechniken auf. Lerner, die ohne spezielle Techniken lernen, erleben das Lernen häufig als ineffizient und anstrengend, was die Lernmotivation substanziell dämpfen kann. Zweitens berichteten insbesondere ältere Beschäftigte eine geringe Gedächtniserwartung, also mangelndes Zutrauen in die Leistungsfähigkeit ihres Gedächtnisses. Geringe Gedächtniserwartung erhöht die Lernangst und führt dazu, dass Lernanstrengungen vorzeitig abgebrochen oder gar nicht erst aufgenommen werden.

Das Training sollte die individuelle Lernorientierung verändern: durch den Aufbau von Änderungsmotivation und eine kognitive Umstrukturierung als Kernintervention. Im ersten Termin standen die Analyse der individuellen Lernmotivation und des Lernmodells im Vordergrund. Haupt- 
punkt der zweiten Sitzung war die Information über alterskorrelierte Veränderungen der Lernkompetenz. Die Sitzungen drei und vier waren der Überprüfung und Korrektur des eigenen Lernmodells gewidmet sowie der Aktivierung von Lernressourcen. Das Ergebnis hat alle Beteiligten überrascht: Innerhalb von vier Wochen konnte das Vertrauen der Trainingsteilnehmer in ihre Lernfähigkeiten erheblich gesteigert werden. Einen solchen Zuwachs hätte vermutlich niemand vor Beginn der Trainings für möglich gehalten.

Die Evaluation vier Monate später erbrachte den objektiven Erfolgsnachweis. Im Vergleich mit einer Kontrollgruppe aus 18 Nicht-Teilnehmern wies eine Stichprobe aus 23 Teilnehmern der Trainingsgruppe bei vergleichbarer Lernleistung eine um ca. 20 \% kürzere Lernzeit auf. Die Beschäftigten, die an den Trainings teilgenommen hatten, ließen eine höhere subjektive Sicherheit beim Lernen sowie günstigere Lerneinstellungen erkennen und sahen weniger Verbesserungsbedarf. Ihr Lernverhalten hatte sich deutlich verbessert. Der Zeitabstand zwischen Training und Evaluation zeigt, dass die Verbesserungen des Lernverhaltens keine kurzfristigen Stimmungseffekte waren, sondern auf tragfähige und längerfristige Verbesserungen hinweisen.

Voraussichtlich lässt sich die Trainingswirkung noch erhöhen, wenn die Randbedingungen der Trainings verbessert werden. Viele der Teilnehmer absolvierten das Training am Schichtende. Eine andere zeitliche Platzierung, z. B. vor Arbeitsbeginn, könnte für bessere Aufnahmefähigkeit sorgen und die Trainingswirkung optimieren. Außerdem legten Gespräche mit Teilnehmern und Unternehmensvertretern nahe, dass die absolute Freiwilligkeit der Teilnahme und die Sicherung eines „geschützten Raums“ Erfolgskriterien sind. An den Trainings sollten keine internen oder externen Beobachter teilnehmen. In einem geschützten Raum, der besonders für ältere Beschäftigte relevant ist, sind die Teilnehmer bereit, Lernprobleme und Unterstützungsbedarfe offen anzusprechen. „Halböffentliche“ Trainings erhöhen hingegen die Hemmschwelle, sich zu artikulieren.

Eine weitere Möglichkeit, die Lernprozesse effizienter zu gestalten, ist die systematische Zusammenstellung der Trainingsgruppen. Im Projekt wurde mit existierenden Arbeitsteams aus der Produktion gearbeitet. Dadurch unterschieden sich die Gruppenmitglieder in ihren Lernkompetenzprofilen teilweise deutlich. Bei manchen Teilnehmern bedurften eher die Lerneinstellungen der Modifikation, bei anderen stand die Erweiterung der Lernstrategien im Vordergrund. Dies begrenzte die Individualisierbarkeit und Effektivität des Trainings. Grundsätzlich lassen sich homogene Trainingsgruppen rekrutieren, mit denen effektiver gearbeitet werden kann, weil alle Gruppenmitglieder ähnliche Arbeitsziele verfolgen.

\section{Zwischenfazit}

Michelin berücksichtigt bei Arbeitsgestaltung und Kompetenzentwicklung bereits die mittel- und langfristigen Auswirkungen des demografischen Wandels und begegnet ih- nen proaktiv. Das Projekt aber zeigt: Lernkompetenz und -motivation auch älterer Beschäftigter lassen sich mit überschaubarem zeitlichem und finanziellem Aufwand deutlich und nachhaltig verbessern.

Gleichwohl ist nicht davon auszugehen, dass die Lernkompetenz beständig hoch bleibt, wenn für die langfristige Sicherung von Lernkompetenz nicht Sorge getragen wird. Dies liegt im Wesentlichen daran, dass Lernkompetenz zwar einerseits Fertigkeiten einschließt (nämlich bestimmte Lernstrategien), die auch längerfristig erhalten bleiben können. Darüber hinaus umfasst Lernkompetenz aber auch Motivationskomponenten, z. B. in Form der Lerneinstellungen, die für die Bereitschaft zur Nutzung der Lernfertigkeiten entscheidend sind. Gerade die Lernbereitschaft aber und mit ihr die Lernkompetenz - können in einem wenig lernförderlichen Umfeld schnell wieder zurückgehen.

Deswegen ist hohe Lern- und Veränderungskompetenz nicht allein Sache der Beschäftigten, sondern eines lernförderlichen Umfelds. Hier kommt der Arbeitsgestaltung eine zentrale Rolle zu, sowohl auf der technisch-objektiven als auch auf der sozialen Ebene, die sich zum Beispiel in Teamstrukturen niederschlägt.

Festzuhalten ist die Eignung der Trainings für kleine und mittlere Unternehmen (KMU). Die Eingangsbefragung im Rahmen der Bestandsaufnahme nahm pro Teilnehmer etwa 20 Minuten in Anspruch, für die Trainings fielen sechs Stunden an, verteilt auf vier Termine à 90 Minuten in Gruppen von max. 15 Teilnehmern und im Abstand von je einer Woche. Dadurch konnten die Trainings in den laufenden Betriebsablauf integriert werden. Dies ist insbesondere für KMU ein wichtiger Faktor, weil die aufwendige Organisation externer Workshops entfällt.

Hervorzuheben ist schließlich die Bedeutung niedrigschwelliger Voraussetzungen für die Trainings. Informations- oder Medienkompetenzen, die nur hochqualifizierte Beschäftigte erfüllen können, würden wichtige Zielgruppen ausschließen. In der Teilnehmergruppe waren zahlreiche Beschäftigte ohne Berufsabschluss oder mit nichtdeutscher Muttersprache. Deswegen wurden Trainingsmaterialien im Papierformat eingesetzt, computerbasierte Trainingsmedien sind folglich nicht notwendig, wenngleich möglich. Der Trainingsansatz eignet sich für den Einsatz mit allen Beschäftigtengruppen und ist leicht auf andere Unternehmen übertragbar.

\section{Nachhaltigkeit fördern: Lernförderliche Arbeitsplätze und Führungskräfteentwicklung}

Selbst in einem Unternehmen wie Michelin sind die Nachhaltigkeitspotenziale nicht ausgeschöpft. Das Unternehmen ist hinsichtlich Trainingsklima und Führungskräfteunterstützung bereits gut aufgestellt. Es kann Lernen aber noch effektiver und nachhaltiger fördern, wenn ein positives Lernklima für alle Altersgruppen geschaffen und erhalten wird.

Dazu sind zwei Entwicklungsziele vordringlich: die lernförderliche Gestaltung der Arbeit und die Führungskräfte- 
entwicklungen mit Blick auf Lernkompetenzen der Beschäftigten. Die gezielte Vermittlung von Informationen über Lernpotenziale und Lernbedürfnisse insbesondere der Älteren an Beschäftigte und Führungskräfte ist gleichermaßen wichtig. Sie verhindert, dass der sich andeutende Altersunterschied im Lernklima sich zu zwei getrennten Klimata auswächst - einem positiven Lernklima für Jüngere und einem negativen für Ältere.

Vor diesem Hintergrund entwickelt Michelin in Bad Kreuznach Strategien, die die Zusammenarbeit und Produktivität altersgemischter Teams verbessern. Im Februar 2014 wurde das Modellprojekt "Altersgemischte Teams“ in der Abteilung Instandhaltung aufgelegt. Dort arbeiten $24 \mathrm{Be}-$ schäftigte im Schichtbetrieb in altersgemischten Teams. Die Abteilung hat gute Produktivität und einen geringen Krankenstand. Alle Beschäftigten verfügen über die objektiven Qualifikationen und Voraussetzungen hoher Leistungsfähigkeit.

\section{Gestaltung lernförderlicher Arbeitsplätze}

Die Lernförderlichkeit der Arbeit soll gezielt gestaltet werden, z. B. indem die Variabilität der Arbeit erhöht wird. Dies kann z. B. durch regelmäßigen Wechsel von Funktionen am Arbeitsplatz, der Kommunikation und Kooperation (z. B Erfahrungsaustausch von Mitarbeitern und Vorgesetzten) und Erhöhung der Partizipation geschehen. Vielversprechende Instrumente sind vorhanden, aber bislang wenig verbreitet. Es geht um vergleichsweise einfache Maßnahmen, wie die Bildung von Projektgruppen aus dem Kreis der Beschäftigten. Sie erhalten den Auftrag, den eigenen Arbeitsbereich hinsichtlich seiner Lernförderlichkeit zu bewerten und Verbesserungsmöglichkeiten zu erarbeiten (z. B. Job Rotation, Aufgabenerweiterung, Vorschläge fürs Weiterbildungsprogramm).

Zwei Überlegungen wurde besondere Aufmerksamkeit geschenkt:

- Innerhalb der altersgemischten Teams existiert ein altershomogenes „40+-Team“. Dieser Konstellation liegt zugrunde, dass einige Abteilungsmitglieder altersbezogene Einstellungen vertreten, die die Zusammenarbeit potenziell ungünstig beeinflussen. Erfahrungsgemäß sind in altersgemischten Teams nicht selten Einstellungen zu finden - z. B., dass jüngere Beschäftigte ältere für weniger lern- und veränderungsbereit halten -, die die Produktivität verringern. Dies kann schlimmstenfalls dazu führen, dass sich bei Älteren eine dauerhafte Leistungsminderung verfestigt.

- Ein zweiter Punkt betrifft den Wissenstransfer. Vorhandene Systeme zur Wissensdokumentation wurden nur relativ gering genutzt. Folglich wird auch das Erfahrungswissen der älteren Beschäftigten weniger verwendet. Wenn dem entgegengewirkt werden soll, muss die Kommunikation in den Teams gut funktionieren. Dies setzt aber angemessene altersbezogene Einstellungen voraus. So kann „Wissenshortung“ vermieden werden.
Vor diesem Hintergrund werden in einem nächsten Schritt altersbezogene Einstellungen der Abteilungsmitglieder ermittelt. Darüber hinaus ermittelt das Projekt Bedarfe der Kompetenzentwicklung im Wissenstransfer. Der Übergang zwischen motivationalen und fertigkeitsbezogenen Gründen für geringe Beteiligung am Wissenstransfer ist häufig fließend. Insbesondere geringes Vertrauen in die eigenen Fähigkeiten, das eigene Erfahrungswissen vermitteln zu können, gründet auf einem Geflecht aus leistungshemmenden Einstellungen, die den Beschäftigten meist selbst wenig bewusst sind, und wenig entwickelten Teilfertigkeiten.

Die Größe der Abteilung erlaubt das Gespräch mit allen Beschäftigten, was eine tragfähige Informationsgrundlage schafft. Dabei führt ein Moderator Gruppengespräche auf der Basis eines semistandardisierten Leitfadens, der in enger Abstimmung mit Michelin entworfen und an betrieblichen (z. B. Arbeitsorganisation, Führungskräfteunterstützung) und persönlichen (z. B. Alternswissen, Adaptivitätskompetenz) Stellgrößen der Teamarbeit ausgerichtet wurde. Gruppendiskussionen bieten den Teilnehmern die Möglichkeit, ihre Sichtweise einzubringen und ihre Fragen rund um die Teamarbeit „im geschützten Raum“ mit externen Experten zu besprechen. Alleine dies erhöht schon das Bewusstsein um die Möglichkeit, die eigene Arbeitssituation mitzugestalten.

In Workshops sollen die Fähigkeits- und Nutzenüberzeugungen der Teilnehmer gestärkt werden. Sie sollen die eigenen Kenntnisse und Stärken bei der Arbeit optimal einbringen können, um nachhaltig arbeiten zu können. Die Workshops befähigen Beschäftigte, nachhaltige Handlungskompetenz aufzubauen, indem sie ihren eigenen beruflichen Entwicklungsbedarf angemessen einzuschätzen lernen, Weiterbildungsangebote effizient nutzen und Entwicklungsbedarfe mit Führungskräften zielgerichtet klären lernen.

Voraussichtlich werden die Workshops für die Beschäftigen eine gewisse Umstellung mit sich bringen. Deshalb ist eine Begleituntersuchung für die ersten beiden Monate vorgesehen. Die leistungsmäßigen, motivationalen, emotionalen Veränderungen werden detailliert abgebildet. Die positiven Effekte lassen sich dadurch „schwarz auf weiß“ rückmelden, potenzielle Barrieren können „zeitnah“ besprochen und damit verringert werden.

\section{Führungskräfteentwicklungen}

Diese Ergebnisse werden Eingang in Führungskräfteentwicklungen finden, um zu verdeutlichen, dass regelmäßige und differenzierte Rückmeldung zum Lernen für Ältere von überproportionaler Bedeutung ist. Vielfach glauben Führungskräfte, Ältere in dieser Hinsicht weniger eng führen zu müssen, weil „die alten Hasen ja schon Bescheid“ wüssten. Ganz im Gegenteil aber zeigen unsere Erfahrungen, ${ }^{3}$ dass das

3 Vgl. Baron, S./Stamov-Roßnagel, C. (2010): Führungskräfte sind für alle da, in: Personal (62), S. 44-48. 
Vertrauen in die eigene Weiterbildungsfähigkeit bei Älteren deutlich stärker von der Ermunterung zur Weiterbildung durch Führungskräfte abhängt als bei Jüngeren. Sie sind also auf regelmäßige Rückmeldung besonders angewiesen.

Gespräche mit Führungskräften zeigen, dass diese oft fürchten, durch mehr Partizipation „schlafende Hunde“ zu wecken, weil den Beschäftigten dann Mängel des eigenen Arbeitsplatzes bewusst oder Begehrlichkeiten geweckt würden, die nicht erfüllt werden könnten. Tatsächlich aber führt die Auseinandersetzung mit der eigenen Arbeit erfahrungsgemäß einerseits zu einem differenzierteren Bild, das „utopische Wünsche" vermindert, andererseits erhöht die Einbindung in die Gestaltung des eigenen Arbeitsplatzes dauerhaft die Motivation und Leistungsbereitschaft. Deshalb lohnt sich Arbeit an der Lernförderlichkeit der Arbeitsgestaltung.

\section{Nachhaltigkeit und die Humanisierung der Arbeit}

Beide Projekte beanspruchen nicht, die „Humanisierung der Arbeit" neu zu erfinden. Aber sie revitalisieren Ideen der Humanisierungsdebatte, die im Laufe der Zeit verschüttet oder vergessen waren. Zum Beispiel die Idee der Gestaltung lernförderlicher Arbeitsplätze. Revitalisierung bedeutet in diesem Zusammenhang, dass zentrale Ideen des Humanisierungsdiskurses mit Blick auf die Herausforderungen der Gegenwart neu konzipiert werden - zum Beispiel jene von älter werdenden Belegschaften und die Aktivierung des Lernpotenzials tendenziell aller Beschäftigter.

Darüber hinaus kamen und kommen zwei weitere Aspekte in diesem Projekt zum Tragen. Erstens, an den Lerntrainings beteiligten sich nicht in erster Linie qualifizierte Facharbeiter, sondern vor allem Beschäftigte mit vergleichsweise geringen Qualifikationen und mit Migrationshintergrund. Dies stellt eine Ausweitung der Klientel der Humanisierungsdebatte dar.
Zweitens stehen nicht allein Ziele und Instrumente im Vordergrund, sondern auch die Absicht, sie zu erreichen und wirksam werden zu lassen. In einem Land mit so entwickelten industriellen Beziehungen wie Deutschland umfasst die Geschichte der Reformen auch die der Reformruinen. Indem der Nachdruck auf nachhaltige Wirkungen gelegt wird, wird auch zum Ausdruck gebracht, dass die nur punktuelle Intention, gute Projekte für gute Zwecke zu machen, nicht mehr ausreicht.

Darüber hinaus gibt es eine erkennbare Chance, organisatorische Innovationen nicht nur in großen Unternehmen mit ihrem großen Potenzial an Steuerungsmöglichkeiten zu realisieren. Wenn Kommunikations- und Kooperationschancen effektiv und effizient genutzt werden, eröffnen sich auch in Klein- und Mittelbetrieben (KMU) Chancen, innovatives Organisationskapital zu entwickeln.

\section{AUTOREN}

CHRISTIAN STAMOV-ROßNAGEL, Prof. Dr., lehrt Organisationspsychologie an der Jacobs University Bremen. Arbeitsschwerpunkte: Förderung individueller Lernfitness, speziell bei älteren Beschäftigten, Entwicklung von Führungskräften in Richtung Demografiemanagement.

c.stamovrossnagel@jacobs-university.de

KLAUS-W. WEST, Dr., ist seit 2010 Geschäftsführer der Chemie-Stiftung Sozialpartner-Akademie (CSSA) in Wiesbaden. Arbeitsschwerpunkte: Wirtschaftsethik ("Wittenberg-Prozess“), Modernität der Industrie und Gestaltung des demografischen Wandels.

klaus.west@cssa-wiesbaden.de 